\title{
A philosophical analysis of neuroenergetics
}

\author{
Robert G. Shulman* \\ Molecular Biophysics and Biochemistry Department, Magnetic Resonance Research Center, Yale University, New Haven, CT, USA \\ *Correspondence: robert.shulman@yale.edu
}

This article represents an opinion by Robert Shulman, who as all know is one of the most senior scientists in the field of neuroenergetics, having made major contributions to field of NMR applied to biology. Robert Shulman is also a deep thinker in the exploration of the relationships between brain activity and mental life. We feel that this personal view on the role that metabolic and vascular imaging as a reflection of neuronal activity may bring to the understanding of mental life, together with a critical appraisal of the limitations of such techniques, is of interest to the field. Hopefully this opinion will stimulate further reflections from readers interested in this topic. Comments of the readers on this essay would be most welcome.

Pierre Magistretti, Specialty Chief Editor, Frontiers in Neuroenergetics

Recent advances in imaging have encouraged neuroscientists to investigate previously unanswerable questions about brain function. Scientists from the many disciplines of neuroscience-psychology, computer science, linguistics, neurochemistry, and cognition - are designing imaging experiments intended to explore their views of brain activities. Because the images measure glucose and oxygen consumption and the rate of blood flow that supplies these nutrients, the experiments track the traditional physiological parameters of brain energy consumption and metabolism. However the experimental possibility of measuring changes in brain properties during behavior such as the response to cognitive tasks, sensory stimulation, and the remembrance of events and instructions, have encouraged studies of mental processes considered to underlie these behaviors. Views of mental processes are not only diverse, but their study raises questions about reliability that long ago were settled in physical chemistry. For example, if I want to talk about my forgetfulness with my wife as in "My memory is failing - I forgot that I was supposed to play poker last night," memory is a very useful concept. However modern imag- ing experiments raise the question as to whether experiments designed to measure rates of glucose consumption that explain "memory" are as reliable as experiments that measure glucose incorporation into glycogen. Questions about the meaningfulness of the different kinds of experiments allowed by imaging chemical reactions in the body can be illustrated by comparing two recent applications of these methods.

\section{PHYSICAL STUDIES OF DIABETES}

Studies of Type 2 Diabetes and the brain responses during a memory task both measure chemical reactions of glucose a common source of human energy but, it will be seen, interpret this information very differently. Type 2 Diabetes has been around - and has been observed - for thousands of years. The great Indian physician Sushruta (fl. sixth century BCE) identified the disease (Stephen et al., 2001) and characterized it by ants being attracted to the urine of patients. Now, after 2000 years of study, we identify the disease by the patient's high blood glucose and by his slow return to normal blood sugar levels after a glucose infusion. We know of the damages wrought by the high glucose and recent studies using magnetic resonance spectroscopy (MRS) have shown that its immediate cause is down regulation of the insulin control of glucose storage as muscle glycogen (Shulman and Rothman, 2001). These metabolic results are one step in the changing understanding of this disease. Our scientific understanding uncovers layers of observables - from the sweet smell that once identified the disease to the present biochemical mechanisms contributing to the high blood glucose. There is in this typical research history not a single step but an unveiling of mechanisms that with time have moved the level of enquiry to the molecular level. In the case at hand of diabetes we now understand the biochemical conditions which cause the disease and allow us to control its symptoms. Clearly it had not been possible until very recently to explain this disease at a molecular or cellular level but from the very beginning of its his- tory its defining properties were observable and measureable. The story of our unfolding analysis, understanding, and control of Type 2 Diabetes is one of the triumphs of the scientific method.

\section{METHODS FOR BRAIN STUDIES}

The road to understanding brain activity has, like our path to understanding Diabetes, been much traveled with advances made possible by methodological discoveries. Long before the nineteenth century insights by physiologists, and the subsequent elaboration of neurons, axons, and synapses we have records from the beginning of the sixteenth century when for a brief period autopsies were allowed in Florence. Leonardo, whose continuing interests in brain anatomy had been interrupted by a temporary ban on the study of cadavers, returned to study the brain's ventricles. Studies of peripheral nerve connections to the ventricles since Galen's time had been inconclusive because the ventricles collapsed when the brain drained upon being excised. da Vinci's (1992) beautiful drawings of the preserved ventricles were made possible by casting techniques he brought with him from his ambitious plans for a horse statue. He filled the ventricles with a soft wax that flowed at a warm temperature and which solidified when the temperature was lowered. The cast of the brain provided by this innovative method disproved the existence of neuronal connections to the ventricles which had been believed for more than a 1000 years.

At present and in conjunction with other approaches in neuroscience, progress in brain studies is being fueled by the chemical and metabolic information provided by non-invasive imaging methods. Noninvasive methods of fMRI, MRS, and PET are responsible for a stunning story of our growing ability to look into the complexities of brain chemistry and physics. In contrast to the muscle, liver, heart, and kidney, all of which can be excised from the body and maintained in a living state on the bench top, the brain must be studied in the living 
person. To this end the possibilities of noninvasive studies of brain activity in vivo have created a wave of excitement in neuroscience, and rightfully so.

Measurements of the cerebral flow of blood and metabolism showed that the brain obtained energy and synthesized metabolic products by oxidizing glucose. These non-invasive methods, in conjunction with other approaches have made it possible to measure and localize cerebral metabolites, thereby allowing us to directly follow metabolism in humans. These developments have created a wide range of possible directions for neuroscience. In addition to metabolic studies of the brain, in which results at the molecular level are similar to those responsible for our present understanding of Type 2 Diabetes, neuroimaging research has also turned to studying mental processes like "memory" or "consciousness." There are thousands of reports on efforts to localize brain regions responsible for mental processes whose origins are to be found in many disciplines particularly psychology although rapidly being extended to the social sciences like economics and political science.

This article will focus on the similarities and differences of scientific method between these two kinds of studies - of the metabolism responsible for Type 2 Diabetes and of the brain participation in mental processes. While it will focus on the uses and misuses of non-invasive investigations of brain function and metabolism, it is also a more basic story of how we posit and pursue questions in scientific research. It is about observation and modest assertions - meaning that it is meant to be a celebration of what we can accomplish in the conduct of observation, experimentation, and analysis, and the danger of allowing $a$ priori assumptions to have undue influence on the conduct of our science. Novel brain results from non-invasive methods spark the temptation to reach further - to assume that our research allows us insight into consciousness or other mental or psychological concepts that make us the human beings that we are. I will ask how we have gone from measurements of brain chemistry to assertions about the mind, the soul, and to ask whether chemistry should seek to explain such matters as how we vote on tax-reform.

\section{MY PATH INTO NEUROSCIENCE}

As a biophysicist I have been enthusiastic about physical studies that can reveal the molecular or cellular mechanism of biological processes - that is what biophysicists and physiologists do. The achievements of physiology testify the successes of this method - the energy consumption of muscle work, the circulation of blood and the understanding of diseases provide explanations and opportunities for control at the molecular level. The surging discoveries in molecular biology supported by novel non-invasive techniques for studying brain activities have encouraged neuroscientists to add brain function and psychological processes to the topics that can be studied at the molecular level. The question for the practicing scientist becomes - what are the criteria for selecting biological phenomena that can be studied with the expectation of finding explanations at the physical-chemical level? I have come to my own answers about these questions only after decades of research, and some notable wrong turns.

I had been doing magnetic research since the 1950s and in the early 1990s, when using MRS to study neurophysiology, I took an excursion offered by the discovery of fMRI (Kwong et al., 1992; Ogawa et al., 1992) which showed that localized changes in brain activity in response to sensory stimulation, such as shining light in the eyes, could be detected. Soon after Seiji Ogawa's demonstration of fMRI with Kamil Ugurbil, both he and Kamil, former colleagues from my years at Bell Labs, came to our Yale laboratory to extend their original measurements on our NMR equipment which Andrew Blamire and Douglas Rothman had modified so as to do fMRI experiments (Blamire et al., 1992).

At this time the field was ablaze as reports accumulated of fMRI responses to various stimuli in numerous laboratories, including reports of brain activities during cognitive tasks like remembering a room or executing a numerical task. In that heady atmosphere where it seemed that mysteries of the mind, in the category of memory and consciousness, were being revealed as regional brain activations, I asked a knowledgeable colleague what well-established mental activity we could study on our equipment that had already measured brain responses to light. My colleague, Patricia Goldman-Rakic, suggested that since short-term memory (sometimes called "working memory") had been identified by invasive electrodes in a specific brain region in non-human primates, we might look for an analogous response in the human brain. The reigning psychological paradigm of cognitive neuroscience assumed that a particular region would be specifically and exclusively motivated by a single activity - in the case at hand, working memory. We did the experiment and found that a particular frontal region indeed was active when the person was doing a task that required remembering, and we published the results (McCarthy et al., 1994).

However, in the excitement of finding a localized brain response to a psychological concept we had been too excited to subject the important claims of cognitive neuroscience to the crucial test of whether the localized response was unique. Our subsequent, more careful, controlled experiments showed that the same region was activated when the subject merely paid attention to a stimulus that did not require remembering (Shulman, 1996). These results, which tested the assumption that there was a unique region responding to what was called, "working memory," contradicted the psychological model. The upshot of these experiments was that the same region was activated during a task requiring attention (and many other activities) without the person being required to remember an earlier event (Shulman et al., 2002). Since the regional activation was not a sufficient response to remembering as postulated by cognitive neuroscience, in order to understand what brain activity could tell us about psychological activities it seemed necessary to unpack the assumptions and findings of cognitive neuroscience. The results of this endeavor, proposed in 1996, (Shulman, 1996) are summarized in this article.

These experiments and subsequent studies made it evident that we are working within assumptions about understanding when we use physical measurements to identify psychological processes in the brain. When studying cerebral metabolism and energetics, as in the synthesis of glycogen in Type 2 Diabetes, it had not been necessary to re-examine epistemological assumptions about the scientific method. 
However, in studying topics like working memory we are not just measuring metabolic responses to clearly defined sensory stimulations by light, sound, or odors; we are not counting beans, we are trying to look at activities of the mind. People have many ideas, opinions, and words to describe the mind, or to decompose it into components like working memory, that are so commonly used they seem to be obvious facts about the world. Like many others, I was eager to locate brain activities that supported our mental life. But as our experiments showed, "working memory" was not being located in the world. Instead it seemed to be an assumption, an hypothesis about brain activity - an hypothesis that was not supported by the experiments. The many reports of cognitive concepts followed by fMRI showed that our results were typical of experimental reports in this active field, in that attempts to identify unique brain locations supporting a concept have failed (Fodor, 2000; Shulman et al., 2002; Friston, 2009). Most directly these results raise the question as to how studies of mental processes differ from scientific investigations capable of explaining more material processes such as Type 2 Diabetes.

\section{POWERS AND LIMITS OF PHYSICAL EXPLANATIONS}

From the early empirical refining of metals to today's understanding and control of man-made materials we can trace the advances in the physical and chemical understanding of non-living phenomenon. The highly developed understanding of the inorganic world has continuously been extended to the living world of plants and animals including humans. Our subject is part of this great historical movement in which studies are broadening to explain the physics and chemistry involved in human phenomenon such as health and behavior. Applications of the laws of physical science to biological molecules like proteins and DNA have encouraged beliefs that we are only at an early stage of applying these laws to living processes. Given the rapid progress in modern biological research directions such as generated by genetics, computer data handling, and brain imaging it is difficult to imagine what limits exist on the understanding that can rightfully be expected from biophysical research.
When we come to the issue at hand, as to how far physics and chemistry can explain the workings of the human brain, we can profit from the analyzes offered by history. The excitement felt today is a continuation of the innovative experiments in the nineteenth century by Claude Bernard and Hermann von Helmholtz who laid the foundations of the physiology we are extending. Bernard proposed methods for physical and chemical explanations of bodily processes by insisting that the object of scientific research is the same for living bodies as for inorganic bodies; it consists in finding the relations that connect any phenomenon with its immediate cause, or putting it differently, it consists in finding the conditions necessary for the appearance of the phenomenon" (Bernard, 1957a). To identify these connections Bernard proposed that phenomena be disassociated into simpler phenomena until finding "conditions that cannot be analyzed (further) in the present state of science": in other words into chemical or physical processes or mechanisms. The goal was to discover the necessary chemical or physical mechanism that caused a phenomenon, and in this way we would understand as well as possible how processes occurred. However, he continued, "The nature of our minds leads us to seek the essence or the why of things. Thus we aim beyond the goal that it is given us to reach, i.e., for experience soon teaches us that we cannot get beyond the how... the immediate cause or the necessary conditions of phenomena. In this respect the limits of our knowledge are the same in biological as in physical-chemical sciences" (Bernard, 1957b).

In neuroscience brain activities are traditionally disassociated into elementary electrical, chemical, and structural processes. When brain activity is described at the electro-chemical level of ion movements across membranes or at the physical level of energy consumption it is, for the present purposes, not reducible further, because the chemical flux of ions and brain energy are in today's science, the ultimate explanations in chemical-physical terms - the hope of biological explanations. To reach this resting point, where no further break down is needed, we have to decompose the general description of activities attributed to the brain into these components. Thus we can find the conditions necessary for the phenomenon of brain activity to take place. We have reached the ultimate in understanding brain activity when it is expressed in its different components of action potentials and energy consumption which are describable in measurable chemical terms of the phenomenon. That understanding is not of the original descriptions of the phenomenon, which was the kind of brain activity that could be identified by lesions or neuroimages, but it was that activity reduced to more physically explainable mechanisms. To describe how a phenomenon happens at the physical-chemical level is the goal of the starting observations. The conditions that are necessary for the phenomenon to occur explains how it happens, and additional studies at the electro-chemical level can amplify our understanding by connecting it with other physical processes but they do not describe why it happens. When we try to describe why something happens we are hoping to have understood it at a deeper level, where the explanation is complete and is uniquely responsible for the event. In arguing that we cannot go beyond physic-chemical explanations Bernard was addressing the vitalist's views that there were fundamental forces such as the élan vital whose explanatory powers provided a more fundamental explanation of why bodily processes occurred. In denying the possibility of explaining why something happens, Bernard was fighting a recurrent position in history. Newton's laws of gravitation were dismissed by some philosophers in his day as having merely provided an explanation of Kepler's laws in terms of a physical law of gravitational attraction, and were criticized for not having provided an explanation of why two bodies attracted each other with a force of gravity.

In looking for an explanation of human behavior by brain processes we are limited to processes that provide necessary support for behavior. Physical experiments can find necessary explanations at the chemical or physical level, but they cannot justify these explanations by appealing to a higher authority because there is no higher authority than physical and chemical explanations. When brain activations are attributed to psychologically postulated mental processes such as consciousness, 
memory, or intention, which are postulated to underlie behavior, instead of to the observed behavior, these experimental results are presumed to tell us why the individual responds to stimuli, or remembers or intends to perform an act. It is possible to find brain activities necessary for an observable human performance: a functioning medial temporal lobe is necessary for a person to remember events and, as we shall see, a high level of brain energy consumption is needed for a person to be in a state of consciousness. On the other hand by postulating that a mental activity called "working memory" is responsible for a person remembering something we are sacrificing a connection between observables (a particular pre-frontal brain region becomes more active when the person is performing a particular act) for a less reliable theory of mental activities. Instead of treating the brain as a material body, subject to physical laws, studies of postulated mental processes like working memory serve psychological ideas about why chemical brain processes occur. In doing so, scientific method is led astray by abstract concepts - powerful ones - where contingent hypotheses, in this case from psychology, are taken as having substantive and discreet reality.

\section{PRAGMATISM AND THE MEANING OF WORDS}

When we do an experiment that looks for the brain location of working memory, it becomes evident that how we theorize about and name a phenomenon - such as "working memory" - is an important framework within which brain science all science - works. Fortunately, in contrast to the philosophers and psychologists who assume they know what is meant by mental processes like working memory, there are other philosophers who caution us about the use of words - proposing that words only have meaning to the extent they influence action. These pragmatists advise us not to assume we actually understand something simply because we have a word for it.

The pragmatist philosopher William James (1995) wrote how it is a mistake to look to words to help understand mysteries:

You know how men have always hankered after unlawful magic, and you know what a great art, in magic, WORDS have always played, If you have his name or the formula of incantation that binds him you can control the spirit, genie, afriete, or whatever the power may be.

... So the universe has always appeared to the natural mind as a kind of enigma. Of which the key must be sought in the shape of (an) illuminating or power-bringing word or name. That word names the universe's PRINCIPLE and to possess it is, after a fashion, to possess, the universe itself. "God," "Matter," "Reason," "The Absolute," "Energy," are so many solving names. You can rest when you have them. You are (at) the end of your metaphysical quest. But if you follow the pragmatic method, you cannot look on any such word as closing your quest. You must bring out of each word its practical cash-value, see it at work within the stream of your experience. It appears less as a solution, than as a program for more work, and more particularly as an indication of the ways in which existing realities may be CHANGED (ITALICSAND CAPS ARE JAMES'S).

What I understand James to be saying is that the existence of a word does not solidify a concept. We have not defined "truth" or "memory" just because we have words for them. A concept like "working memory" is defined by the actions its leads to and therefore is intrinsically dependent upon its specific usage or the context in which it is identified. When we claim working memory is found in a person who is remembering recently observed numbers or faces or the third last word he saw we are not starting with the observation of a general property found in all cases, like the sweet smell of urine identifying diabetes, but are proposing there is a general concept that underlies all these observations. However a word is not a specific tangible creation; it is an hypothesis that a person finds useful when dealing with the world. We can profitably use a word like memory to describe how well something is remembered by a person or group, (or whether I am habitually forgetting my poker dates) but it is a long stretch to assume that the word memory used to describe a student reciting the multiplication table is the same thing as the word that Marcel Proust used to describe what was evoked by the smell of madeleines. It is difficult to see how reliable empirical brain activities, like the neural correlates of consciousness, or activations of specific brain regions in an $\mathrm{fMRI}$ experiment, might be found for such a word. And, in fact, experimental fMRI results do not find a single reproducible response for a term such as working memory but rather find different brain regions responding that depend upon the context in which the word is used (Fodor, 2000; Shulman et al., 2002; Friston, 2009). These results are in agreement with the pragmatist's view that there is no substance to the word other than in the actions it generates.

Why do I claim that biological processes like the immune response, genetics, and metabolism, can be explained in physicalchemical terms while mental processes like memory and consciousness cannot be? The simple answer is that inheritance of traits, the immune response, and metabolism are activities that can be observed and measured while the psychological processes described as memory or intention are not observables but are hypotheses created by theories that help us describe the world.

\section{FRANCIS CRICK'S STUDY OF CONSCIOUSNESS}

The distinction in neuroscience between experiments designed to reduce conceptualizations of biological phenomena to molecules and cells and experiments designed to find molecular and cellular mechanisms of observables can be seen clearly in the studies of consciousness. Consciousness is a central interest today of neuroscientists attracting discussions from its many sub-disciplines. Journals, books, associations, and an annual meeting all specifically address the study of consciousness.

It may come as no surprise that the search for a molecular basis of consciousness has been led by Francis Crick, whose great elucidation of the DNA structure led to the field of molecular biology, the twentieth century revolution in biology. In 1994 Crick focused the study of consciousness when in his book, The Astonishing Hypothesis: The Scientific Search for the Soul (Crick, 1994a page 7), he stated: "our minds - the behavior of our brains - can be explained by the interactions of nerve cells (and other cells) and the molecules associated with them." Crick defines the terms of his reductionist approach in this way: "a complex system can be explained by the behavior of its parts," and proposes that the reduction needs to go no further than finding explanations at the neural or chemical level using the laws of classical physics. 
In advocating the explanation of consciousness at the neural level he writes that such reductionism is "not the rigid processes of explaining one set of ideas in terms of another fixed set of ideas at a lower level, but a dynamic interactive process that modifies the concepts at both levels as the knowledge develops" (Crick, 1994b). He recognizes that since Descartes the mental and the material have been considered to be separate, incommensurate domains, but claims that this method of explaining a system property at the chemical level does not involve a "category mistake." He acknowledges that the mental and material have been considered to be of different categories and that proposing to explain one in terms of the other has been considered to be a mistake (the familiar body-mind problem), but he claims that recent advances in reductionist studies have enabled science to overcome these categorical differences. In support of this hypothesis, he notes that in earlier days the problem of a reductionist approach "could have taken the form that to consider a gene to be a molecule (or as we should say now, a part of a matched pair of molecules) would be a category mistake. A gene is one category and a molecule is a quite different category. One can see now how hollow such objections have turned out to be" (Crick, 1994c). In my opinion, this statement, made in the optimistic early days of the Human Genome Project asserting that the DNA molecule explained the gene, is misleading in two important ways.

First Crick proposes that the gene which has been explained by DNA is of the same category as consciousness and thereby sets an example for his goal to reduce consciousness to molecules. However the gene, at the beginning of Crick and Watson's studies, had been defined by observations as the fundamental unit of inheritance (Encyclopedia Britannica, 1932). The properties of genes were observables - Mendel's pea shoots reflected genes for yellow or green peas while the genes identified in Morgan's fruit flies controlled the inheritance of red eyes and other observable traits. In this important respect the gene differed from consciousness which Crick acknowledged could not be defined by observation. The explication of the DNA structure provides a way to study the processes of inheritance attributed to the gene in molecular terms. The DNA structure became the basis of a new field of study, molecular genetics, which will continue the study of the many steps of inheritance, e.g., meiosis, development, and phenotype expression, in molecular terms (Keller, 2000). In this role DNA actually serves as the beginning of biochemical research into the processes of heredity. The observable inheritance of many physical properties like the red eye in Drosophila now can be explained at the molecular level starting with the DNA sequence just as the observable sweet smell identifying Diabetes now can be explained by the metabolism of glucose. But consciousness is not an observable - it was a concept presumed to underlie a person's behavior. It was of a different category than the gene and there is no reason to believe that advances in biology have suddenly made it understandable in molecule terms. Hence the role of DNA in providing mechanisms for actions attributed to the gene is very different from how Crick proposes to explain that "You, your joys and your sorrows, and your ambitions, your sense of personal identity and free will are in fact no more than the behavior of a vast assembly of nerve cells and their associated molecules" (Crick, 1994d).

My second criticism is that Francis Crick and Jim Watson never studied the gene, although their results have important consequences for understanding the process of heritability. They studied base pairing, hydrophobic interactions of phosphates, $\mathrm{X}$-ray diffraction patterns, and possible structural models of the DNA molecule. Their structural studies of a chemical molecule, that had been known to be involved in genetic processes, changed our understanding of genetics and biology for all time. But they did not study the gene. Although the gene was a description of the observable processes of inheritance those processes had not been described in molecular terms and could not be studied directly. When Crick suggests that molecular studies are a useful method for explaining consciousness he is asking us to do something quite different from what he and Watson did. In brain studies I suggest we should do what Crick and Watson actually did, not what Crick subsequently proposed. They studied the properties of a molecule that previously had been shown to play a necessary role in the observable transmission of genetic information and thereby opened observable, inheritable traits, like the red eyes of Drosophila, to molecular studies.

\section{A DIFFERENT APPROACH TO THE STUDY CONSCIOUSNESS}

In following the successes carved out by Crick and Watson I propose that to explore consciousness we should find a physical, brain activity that is necessary for the person to show the observable activities of consciousness. Studying that physical, molecular activity should, in time, reveal more aspects of a person's activity while in the state of consciousness - just as the DNA structure opened pathways for the study of inheritance. We have proposed how this can be done in a preliminary report (Shulman et al., 2009) sketching a method of studying consciousness that relates a physical property, in this case the high brain energy, to an observable, the person's activities in a state of consciousness, which had not yet been described in molecular terms. The exploration follows upon the investigation of the philosophical foundations of neuroscience, the title of a path-breaking book by Bennett and Hacker (2003). Their main point is that "It is not the brain that is conscious or unconscious, but the person whose brain it is.... One sees with one's eyes and hears with one's ears, but one is not conscious with one's brain." This clarification means that the definition of consciousness depends upon something the person does such as his response to stimuli, and while certain brain activity can be necessary to maintain the person in the state of consciousness the brain does not do consciousness. A person's actions of consciousness, they propose, can be broken into two forms of which we are concerned with the first. Following conventions in the field we call this form the state of consciousness which they define as "a matter of being conscious or awake as opposed to being unconscious or asleep." The state of consciousness is my concern because it a third person observable state. There is no mystery or contingency in saying that a person is in a state of consciousness when we see him responding to stimuli - anesthesiologists do it all the time and their conclusion is the basis of important clinical decisions. My argument is that once a person can be defined as being in the state of consciousness by observing his response to stimuli - as done routinely by the anes- 
thesiologists, we can measure properties of that state, including brain activities. We have identified brain properties characteristic of that state which disappear when the person moves out of that state - as a result of anesthesia or falling asleep of suffering a coma. Experiments, from MRS and PET, show that the high global level of neural energy plays a necessary role in maintaining humans in the observable, behavioral state of consciousness. Several additional brain properties of that state have been reported (Shulman et al., 2009) where the science of this subject is discussed.

In defining properties of the state of consciousness we find an observable role for the total brain energy. Instead of aiming to localize mental processes by postulating mental concepts and localizing these reifications in the brain by small energy changes, (Shulman and Rothman, 2004) our philosophical considerations have brought us to hypothesize that high cerebral energetics is a necessary brain property of the person when in the observable state of consciousness. The MRS and PET data have supported this hypothesis by showing that the global brain energy consumption is reduced by anesthesia, sleep, and coma which bring the person out of the state of consciousness. Our previous experimental studies (Mandaag et al., 2007) have shown that brain energy consumption is a reliable measurement of neuronal activity. Hence these results establish a connection between total, global neural activity, and the observable state of consciousness and open the neural properties of that state to further molecular study.

\section{IN PLACE OF BEHAVIORISM}

In retrospect it is somewhat surprising that functional imaging results, which are neurophysiological measurements of changes in blood flow and glucose oxidation, should from their very beginning be interpreted as evidence for psychological activities (Posner and Raichle, 1994). In place of the methods of cognitive neuroscience based on psychology this article relates empirical brain measurements to observations of behavior, thereby avoiding a role for psychological processes presumed to underlie behavior. By confining attention to brain processes that are necessary for the person to perform the observed behavior, and by not postulating or studying mental processes hypothesized to relate brain activities to the observed behavior, my approach has some similarities with the once popular school of psychology called behaviorism. While there are similarities with that field in our dependence upon observable behavior, our methods still differ significantly from this older psychology as well as from the more recent cognitive psychology. Watson (1932) definitive summary showed the reliable role of behavior in science. Watson started with the clear statement that "Behavior can be observed like the phenomena of all other natural sciences." When controlled experiments established the connection between stimulus and response then, Watson continued, the behaviorist's psychological questions have been answered. Not being able to observe in these investigations any so-called mental processes, like "consciousness, sensation, perception, imagery, or will," behaviorists, Watson writes, "reached the conclusion that all such terms can be dropped out of the description of man's activity." He continued: "the neurologists and physical chemists have problems to solve about the neuronal connections and in determining the physical and chemical work done in the reaction." However, he adds, those are not the concern of psychology, which he felt could be best pursued as the study of behavior. Watson does not conclude that mental processes do not exist, or that the brain played no role in supporting them - quite the contrary - he simply does not think that psychology had the tools to study them. That was close to a century ago. In the 1970s cognitive psychology tried to fill the gap between stimulus and response by proposing both the nature of these mental processes and the brain mechanism for dealing with them from the perspective of computers, information theory, and linguistics. I share Watson's enthusiasm about the reliable explanatory powers of observable behavior and, as a pragmatist, share his skepticism about the value of assuming mental processes. As a modern representative of the "neurologists" and physical chemists to whom he defers, I believe that we physical scientists can pursue the mechanisms of brain activity during a person's behavior, but not by invoking mental processes responsible for that behavior. Freed from the assumption that cognitive neuroscience has answered the questions that behaviorism felt to be beyond the capability of psychology, we have relied upon neurophysiological studies of brain energy and work for insights into neuronal support of observable behavioral activities.

\section{ACKNOWLEDGMENTS}

I thank Douglas L. Rothman and Fahmeed Hyder for continuing discussions of these ideas based upon their experimental results and Maxwell Bennett, Christoph Jurgen, Vin Marchese, James Shulman, Barbara Herrnstein Smith, Robert Turner, and Bruce Wexler for comments on drafts of this manuscript which is adapted from the first chapter of a book on Philosophy of Neuroscience being prepared for publication at Oxford Press.

\section{REFERENCES}

Bennett, M. R., and Hacker, P. M. S. (2003). Philosophical Foundations of Neuroscience. Malden, MA: Blackwell Publishing.

Bernard, C. (1957a). An Introduction to the Study of Experimental Medicine. New York, NY: Dover Publications, 66.

Bernard, C. (1957b). An Introduction to the Study of Experimental Medicine. New York, NY: Dover Publications, 80.

Blamire, A. M., Ogawa, S., Ugurbil, K., Rothman, D. L., McCarthy, G., Ellerman, J. M., Hyder, F., Rattner, Z., and Shulman, R. G. (1992). Functional magnetic resonance imaging of human prefrontal cortex activation during a spatial working memory task. Proc. Natl. Acad. Sci. U.S.A. 89, 11069-11073.

Crick, F. H. C. (1994a). The Astonishing Hypothesis. New York: Charles Scribner's Sons.

Crick, F. H. C. (1994b). The Astonishing Hypothesis. New York: Charles Scribner's Sons, 8.

Crick, F. H. C. (1994c). The Astonishing Hypothesis. New York: Charles Scribner's Sons, 9.

Crick, F. H. C. (1994d). The Astonishing Hypothesis. New York: Charles Scribner's Sons, 3.

da Vinci, L. (1992). The Anatomy of Man: Drawings from the Collection of Her Majesty Queen Elizabeth 11. Boston: Museum of Fine Arts Houston and Bullfinch Press Little Brown \& CO.

Encyclopedia Britannica. (1932). Gene, 14th Edn, Vol. 10 New York, NY: Encyclopedia Britannica.

Fodor, J. (2000). The Mind Doesn't Work That Way: The Scope and Limits of Computational Psychology. Cambridge, MA: MIT Press.

Friston, K. (2009). Modalities, modes and modes in functional neuroimaging. Science 326, 399-403.

Keller, E. F. (2000). The Century of the Gene. Cambridge, MA: Harvard University Press, 155-159.

Kwong, K. K., Belliveau, J. W., Chesler, D. A., Goldberg, I. E., Weiskoff, R. M., Poncelet, B. P., Kennedy, D. N., Hoppel, B. E., Cohen, M. S., Turner, R., Cheng, H. M., Brady, T. J., and Rosen, B. R. (1992). Dynamic magnetic resonance imaging of human brain activity 
during primary sensory stimulation. Proc. Natl. Acad. Sci. U.S.A. 89, 5675-5679.

Mandaag, N. J., Coman, D., Sanganahalli, B. G., Herman, P., Smith, A. J., Blumenfeld, H., Shulman, R. G., and Hyder, F. (2007). Energetics of neuronal signaling and fMRI activity. Proc. Natl. Acad. Sci. U.S.A. 104, 20546-20551.

McCarthy, G., Blamire, A. M., Puce, A., Nobre, A. C., Bloch, G., Hyder, F., Goldman-Rakic, P., and Shulman, R. G. (1994). Functional magnetic resonance imaging of human prefrontal cortex activation during a spatial working memory task. Proc. Natl. Acad. Sci. U. S. A. 91, 8690-8694.

Ogawa, S., Tank, D. W., Menon, R., Ellerman, J. M., Kim, S. G., Merkle, H., and Ugurbil, K. (1992). Intrinsic signal changes accompanying sensory stimulation: functional brain mapping with magnetic resonance imaging. Proc. Natl. Acad. Sci. U.S.A. 89, 5951-5955.
Posner, M. I., and Raichle, M. E. (1994). Images of Mind. New York: Scientific American Press.

Shulman, R. G. (1996). Interview with Robert G. Shulman. J. Cogn. Neurosci. 8, 474-480.

Shulman, R. G., Hyder, F., and Rothman, D. L. (2002). Biophysical basis of brain activity: implications for neuroimaging. Q. Rev. Biophys. 35, 287-325.

Shulman, R. G., and Rothman, D. L. (2001). 13C NMR of intermediary metabolism: implications for systemic physiology. Annu. Rev. Physiol. 63, 15-48.

Shulman, R. G., and Rothman, D. L. (2004). Brain Energetics and Neuronal Activity. Chichester: John Wiley.

Shulman, R. G., Hyder, F. H., and Rothman, D. L. (2009). Baseline brain energy supports the state of consciousness. Proc. Natl. Acad. Sci. U.S.A. 102, 11096-11101.
Stephen, L., Last, J. M., and Dunea, G. (2001). The Oxford Illustrated Companion to Medicine. New York: Oxford University Press.

Watson, J. B. (1932). Behaviorism Encyclopedia Britannica. 14th Edn, Vol. 3. New York, NY:Encyclopedia Britannica, 327.

William James. (1995). Pragmatism: A new name for some old ways of thinking. New York, NY: Dover Press.

Received: 14 April 2011; accepted: 12 September 2011; published online: 18 November 2011.

Citation: Shulman RG (2011) A philosophical analysis of neuroenergetics. Front. Neuroenerg. 3:6. doi: 10.3389/ fnene. 2011.00006

Copyright $\odot 2011$ Shulman. This is an open-access article subject to a non-exclusivelicense between the authors and Frontiers Media SA, which permits use, distribution and reproduction in other forums, provided the original authors and source are credited and other Frontiers conditions are complied with. 